\title{
Heart adaptations to long-term aerobic training in paraplegic subjects: an echocardiographic study
}

\author{
MA Maggioni ${ }^{1}$, M Ferratini ${ }^{2}$, A Pezzano ${ }^{2}$, JE Heyman ${ }^{2}$, L Agnello ${ }^{3}$, A Veicsteinas ${ }^{1}$ and G Merati ${ }^{1,3}$
}

Study design: Case-control.

Objectives: To execute an echocardiographic comparison between trained and untrained spinal cord injury (SCl) subjects, and to evaluate whether long-term heart adjustments to endurance training are comparable to those observed in able-bodied (ABL) subjects. Setting: Italy.

Methods: We enrolled: (1) 17 male SCI patients (lesion level $\mathrm{T}_{1}-\mathrm{L}_{3}, 34 \pm 8$ years, body mass index (BMI) $23.0 \pm 2.8 \mathrm{~kg} \mathrm{~m}^{-2}$ ), 10 of whom were aerobically trained for $>5$ years $\left(\mathrm{SCl}_{\mathrm{T}}\right)$; (2) 18 age-, sex- and BMI-matched $\mathrm{ABL}$ subjects $(35 \pm 6$ years, BMI $23.6 \pm 2.8 \mathrm{~kg} \mathrm{~m}^{-2}$ ), 10 of whom were aerobically trained for $>5$ years $\left(A B L_{T}\right)$. Training frequency and volume were recorded by a dedicated questionnaire. All subjects underwent a trans-thoracic echocardiography; SCI subjects also performed an exhaustive incremental exercise test. Comparisons were made between $\mathrm{ABL}$ and $\mathrm{SCl}$ groups, between trained and untrained subjects within each group (analysis of variance).

Results: Effects of SCl-Compared with ABL subjects, SCl patients showed lower end-diastolic volume (76 \pm 21 vs $113 \pm 23 \mathrm{ml}$, $P<0.05)$ and ejection fraction ( $61 \pm 7 \%$ vs $65 \pm 5 \%, P<0.05)$. Effects of training - Compared with untrained status, the intra-ventricular septum thickness ( $\mathrm{SCl},+18 \%$; $\mathrm{ABL},+4 \%$ ), the posterior wall thickness $(\mathrm{SCl},+17 \%$; $\mathrm{ABL},+2 \%)$ and the total normalized heart mass ( $\mathrm{SCl},+48 \%$; $\mathrm{ABL},+5 \%$ ) were higher in both $\mathrm{SCl}_{T}$ and in $\mathrm{ABL}_{\top}$. $\mathrm{VO}_{2}$ peak was higher in the $\mathrm{SCl}_{T}$ subgroup compared with the $\mathrm{SCl}_{\mathrm{U}}$ group. Conclusions: Heart seems to positively adapt to long-term endurance training in $\mathrm{SCl}$ patients. Regular exercise may therefore increase heart size, septum and posterior wall thickness, which likely contributes to improved $\mathrm{VO}_{2}$ peak. These morphological and functional changes may reduce cardiovascular risk in $\mathrm{SCl}$ individuals.

Spinal Cord (2012) 50, 538-542; doi:10.1038/sc.2011.189; published online 24 January 2012

Keywords: spinal cord injury; training; endurance; left ventricle; echocardiography

\section{INTRODUCTION}

The positive effects of endurance training on heart morphology and function are well acknowledged in able-bodied (ABL) individuals: besides the typical development of bradycardia and the improvement in coronary perfusion, cardiac morphology usually shifts toward a physiological left ventricular (LV) hypertrophy with increased mass and internal volume of the left ventricle, and improved systolic and diastolic functions (for a review, see Pavlik et al. ${ }^{1}$ ). In ABL endurance-trained individuals, the increased stroke volume finally yields an augmented cardiac output during exercise compared with untrained subjects. ${ }^{2}$

A previous study demonstrated a reduction in LV mass and dimension in tetraplegic subjects, ${ }^{3}$ and a more recent study showed an altered LV diastolic function and a subclinical decrease in systolic function in spinal cord injury (SCI) individuals. ${ }^{4}$ In these patients, the reduced venous return due to the loss of sublesional vascular sympathetic innervation and of the muscular pump may cause a reduced adaptation of stroke volume to exercise, ${ }^{5}$ which needs to be compensated by a higher submaximal heart rate, compared with that observed in ABL subjects. ${ }^{6,7}$ Indeed, Dela et al. ${ }^{5}$ demonstrated a stroke volume increase of about $+35 \%$ in paraplegics compared with a $+50 \%$ increase in ABL people during a steady-state moderate exercise. This may limit the cardiac output during physical workout, which would directly relate to a lower $\mathrm{VO}_{2}$ peak.
While the known heart adaptations to endurance training have been confirmed by some proponents of exercise in SCI people, ${ }^{8}$ such adaptations have been questioned by other opponents of exercise. ${ }^{9}$ Gates et al. ${ }^{9}$ found no differences in LV structure and function between endurance- and power-trained SCI athletes compared with sedentary SCI subjects. Moreover, it is still uncertain whether in SCI subjects the exercise-induced modifications of myocardial structure and function can be preserved in the long term by maintaining an adequate level of aerobic physical fitness.

The aims of this study were to compare baseline echocardiographic parameters between SCI and ABL subjects, and to assess whether heart adjustments to long-term training are comparable in SCI and ABL subjects. In addition, we aimed at evaluating the differences in maximal aerobic capacity and the relationship between echocardiographic parameters and maximal oxygen uptake between sedentary and trained SCI individuals.

\section{MATERIALS AND METHODS}

Subjects

We enrolled 17 male SCI patients (lesion level $\mathrm{T}_{1}-\mathrm{L}_{3}$, ASIA Scale A, age $34 \pm 8$ years, body mass index (BMI) $23.0 \pm 2.8 \mathrm{~kg} \mathrm{~m}^{-2}$ ), 10 of whom were aerobically trained $\left(\mathrm{SCI}_{\mathrm{T}}\right)$ for at least 5 years. In addition, 18 age- and BMI-matched $\mathrm{ABL}$ male subjects ( $35 \pm 6$ years, BMI $23.6 \pm 2.8 \mathrm{~kg} \mathrm{~m}^{-2}$ ), 10 of whom were aero-

${ }^{1}$ Department of Sport Sciences, Nutrition and Health, University of Milan, Milan, Italy; ${ }^{2}$ Cardiologic Rehabilitation Unit, Don C. Gnocchi Foundation, Milan, Italy and ${ }^{3}$ Center of Sports Medicine, Milan, Italy

Correspondence: Dr G Merati, Department of Sport Sciences, Nutrition and Health, Via Colombo, 71 University of Milan, Milan 20133, Italy.

E-mail: giampiero.merati@unimi.it

Received 16 June 2011; revised 19 December 2011; accepted 20 December 2011; published online 24 January 2012 
Table 1 Demographic and anthropometric features of the enrolled subjects, divided for pathology and training status

\begin{tabular}{lccccc}
\hline & $S C I_{U}$ & $S C I_{T}$ & $A B L_{U}$ & $A B L_{T}$ & $\mathrm{P}$ \\
\hline$n$ & 7 & 10 & 8 & 10 & \\
Lesion level & $\mathrm{T}_{1}-\mathrm{L}_{3}$ & $\mathrm{~T}_{1}-\mathrm{L}_{1}$ & - & - & \\
Age (years) & $36 \pm 10$ & $33 \pm 7$ & $33 \pm 6$ & $33 \pm 8$ & 0.55 \\
Weight (kg) & $73 \pm 10$ & $70 \pm 8$ & $73 \pm 14$ & $72 \pm 6$ & 0.87 \\
Height (cm) & $173 \pm 8$ & $178 \pm 3$ & $174 \pm 7$ & $177 \pm 6$ & 0.28 \\
BMI (kg m${ }^{-2}$ ) & $24.1 \pm 3.1$ & $22.1 \pm 2.3$ & $24.3 \pm 3.7$ & $23.0 \pm 1.9$ & 0.29 \\
\hline
\end{tabular}

Abbreviations: $A B L_{T}$, aerobically trained able-bodied subjects; $A B L_{U}$, aerobically untrained ablebodied subjects; $\mathrm{SCl}_{\mathrm{T}}$, aerobically trained spinal cord injury subjects; $\mathrm{SCl}_{U}$, aerobically

untrained spinal cord injury subjects. Data are mean \pm s.d. $P$ value (one-way ANOVA)

$P$, significance value from one-way ANOVA.

bically trained $\left(\mathrm{ABL}_{\mathrm{T}}\right)$ for at least 5 years were recruited. None of the subjects was a current smoker and no one had arterial hypertension or diabetes. Other exclusion criteria were the presence of severe cardiac diseases (cardiomyopathies, cardiac failure, moderate to severe cardiac valvulopathies, recent myocardial infarction, ventricular aneurysms), which could limit the cardiac function and/or cause a LV remodeling. The demographic data of the enrolled subjects, stratified according to pathology and training status ( $\mathrm{T}$, trained; $\mathrm{U}$, untrained), are shown in Table 1.

After receiving a full explanation of the purpose of the study and of the experimental procedures, all subjects signed a written informed consent. The study was approved by the ethical committee of the Don C Gnocchi Foundation and performed according to the principles of the Declaration of Helsinki.

Statement of ethics. We certify that all applicable institutional and governmental regulations concerning the ethical use of human volunteers were followed during the course of this research.

\section{Experimental procedures}

Echocardiography. All subjects underwent a trans-thoracic echocardiography (mod. Sequoia Acuson 512, Siemens, Germany, equipped with a $3.5-\mathrm{MHz}$ phased-array transducer). According to the statements of the American Society of Echocardiography Standards, ${ }^{10}$ the following parameters were measured: (1) left ventricular (LV) end-diastolic diameter (EDD) and volume (EDV); (2) intra-ventricular septum thickness (IVST, end diastole); (3) posterior wall thickness (PWT, end diastole); (4) ejection fraction, calculated from the apical four-chamber view as:

$$
[(\mathrm{EDV}-\mathrm{ESV}) / \mathrm{EDV}) * 100]
$$

where EDV is end-diastolic volume and ESV is end-systolic volume; (5) LV mass (LVM), calculated according to the Devereux and Reicheck formula, ${ }^{11}$ and normalized per body surface area:

$$
\mathrm{LVM}=1.04\left[(\mathrm{LVID}+\mathrm{PWT}+\mathrm{IVST})^{3}-(\mathrm{LVID})^{3}\right]-13.6 g
$$

where LVID is diastolic LV internal diameter, PWT is posterior wall thickness and IVST is intra-ventricular septum thickness; (6) peak early inflow velocity $(E)$, peak atrial inflow velocity $(A)$ and peak early/atrial velocity ratio $(E / A) ;(7)$ iso-volumic relaxation time (IVRT), defined as the time interval between aortic valve closure and mitral valve opening, which reflects the rate of LV relaxation. ${ }^{12}$

Incremental exercise test. An incremental exercise test up to exhaustion was executed on a separate day on SCI subjects only. The testing procedure was performed by an adapted wheelchair ergometer (Ergotronic 4000, Sopur, Heidelberg, Germany). The exercise protocol began at an initial velocity of $2 \mathrm{~km} \mathrm{~h}^{-1}$ and continued with 3-min steps, with a speed increment of $2 \mathrm{~km} \mathrm{~h}^{-1}$ per step; the test was stopped at the volitional exhaustion. This protocol is similar to that reported in Hartung et al., ${ }^{13}$ which measured maximal oxygen uptake by steps of $2 \mathrm{~min}$ and increments of $3 \mathrm{~km} \mathrm{~h}^{-1}$. In our protocol we chose to increase the step time and reduce the velocity increment: in this way, oxygen consumption for each step is likely to reach a sufficiently long steady state in the last phase of each step. Three-minute steps on manual ergometers were used by other authors. ${ }^{14}$

Respiratory gases were collected at rest for about $3 \mathrm{~min}$ and during the last minute of each exercise step. The following parameters were measured: heart rate (HR, b.p.m.) by continuous electrocardiographic recording in $\mathrm{V}_{5}$ lead (Cardioline Delta 1 Plus, Milan, Italy), and volumes and $\mathrm{O}_{2}$ and $\mathrm{CO}_{2}$ concentrations in expired air (\% vol; Oxygen Analyser, Servomex, Crowborough, UK, and Binos C, Fisher Rosemount, Wesseling, Germany), collected in 150-1 Douglas bags. Gas analyzers were calibrated before each experiment.

Physical activity questionnaire. All the trained ABL and SCI individuals were athletes referring to the Sports Medicine Centre of the Don C Gnocchi Foundation (Milan, Italy) for pre-participation screening in agonistic activities during the last 5 years. The training duration was therefore retrieved by their individual clinical records: a subject was classified as a 'long-trained endurance athlete' if he had a history ( $>5$ years) of endurance training (for example, long distances in track and field, wheelchair marathon, hand-bike, swimming, Nordic skiing, and so on) at least three times weekly ( $1.5 \mathrm{~h}$ at least for each training session). The actual training status of the subjects was assessed by the localized Italian version of the validated International Physical Activity Questionnaire. ${ }^{15}$ The study participants were classified as 'sedentary' if they were categorized in the 'lowest activity level' of the Questionnaire. The duration of the sedentary status, if any, was finally assessed by a non-validated recall questionnaire on previous recreational/sport activities in ABL subjects.

Based on the questionnaire results, we divided each of the SCI and the ABL groups into two further subgroups: $\mathrm{SCI}_{\mathrm{T}}$ (trained) $(n=10), \mathrm{SCI}_{\mathrm{U}}$ (untrained) $(n=7) ; \mathrm{ABL}_{\mathrm{T}}(n=10)$ and $\mathrm{ABL}_{\mathrm{U}}(n=8)$.

Statistical analysis. If not otherwise stated, results are shown as mean \pm s.d. All parameters were normally distributed (Shapiro-Wilk test) and there were no missing data. The one-way analysis of variance was preliminarily applied to verify the data matching between the four trained and untrained subgroups. A $2 \times 2$ factorial analysis of variance was then used to evaluate the differences in echocardiographic parameters between the four subgroups, and the post hoc least significant difference Fisher test was applied where appropriate. The statistical regression was computed by the least-squares method, and the $r$ coefficient was then calculated.

The level of statistical significance was set at $P<0.05$. Statistical analyses were performed using the Statistical software package Statistica 7.0 (StatSoft, Tulsa, OK, USA).

\section{RESULTS}

The demographic and anthropometric data of the enrolled subjects, stratified according to pathology and training status ( $\mathrm{T}$, trained; $\mathrm{U}$, untrained), were matched between groups (Table 1).

\section{Echocardiography}

SCI vs ABL subjects. To assess the statistical differences in cardiac parameters due to $\mathrm{SCI}$, we pooled $\mathrm{SCI}_{\mathrm{T}}$ and $\mathrm{SCI}_{\mathrm{U}}$ data and $\mathrm{ABL}_{\mathrm{U}}$ and $\mathrm{ABL}_{\mathrm{T}}$ data. SCI patients showed significantly lower EDD (44.3 \pm 5.6 vs $47.5 \pm 5.4 \mathrm{~mm}, P=0.04)$ and $\operatorname{EDV}(76.2 \pm 20.8$ vs $112.9 \pm 22.9 \mathrm{ml}$, $P=0.001)$ than $\mathrm{ABL}$ subjects, respectively. Similarly, the ejection fraction $(60.7 \pm 7.0 \%$ vs $65.3 \pm 5.3 \%, P=0.03)$ was significantly lower in SCI compared with ABL individuals. Surprisingly, the IVST was slightly but significantly higher in SCI subjects $(9.5 \pm 1.3$ vs $8.7 \pm 0.7 \mathrm{~mm}, P=0.02)$, whereas the PWT $(9.2 \pm 1.3$ vs $8.6 \pm 0.7 \mathrm{~mm})$ did not significantly differ, although a trend toward a higher value in SCI group was perceived. The LVM normalized per body surface area was not significantly different between SCI $\left(71.6 \pm 21.0 \mathrm{~g} \mathrm{~m}^{-2}\right)$ and ABL $\left(76.2 \pm 14.8 \mathrm{~g} \mathrm{~m}^{-2}\right)$ subjects. The peak early inflow velocity (E: $0.66 \pm 0.14 \mathrm{~m} \mathrm{~s}^{-1}$ in SCI and $0.71 \pm 0.14 \mathrm{~m} \mathrm{~s}^{-1}$ in ABL), peak atrial inflow velocity $\left(A: 0.48 \pm 0.10 \mathrm{~m} \mathrm{~s}^{-1}\right.$ in SCI and $0.44 \pm 0.07 \mathrm{~m} \mathrm{~s}^{-1}$ in $\mathrm{ABL})$ and peak early/atrial velocity ratio (E/A: $1.56 \pm 0.56$ in SCI and 
$1.66 \pm 0.39$ in $\mathrm{ABL}$ ) did not differ between SCI and ABL groups. Finally, the IVRT was significantly higher in SCI subjects $(103 \pm 8 \mathrm{~ms})$ compared with $\mathrm{ABL}$ individuals $(56 \pm 9 \mathrm{~ms} ; P<0.001)$.

Subgroup analysis in trained vs untrained subjects. The main echocardiographic parameters, stratified according to the training status, are shown in Table 2 . In particular, the $\mathrm{SCI}_{\mathrm{T}}$ subgroup showed higher IVST values and a trend toward an increased PWT compared with the $\mathrm{SCI}_{\mathrm{U}}$ subgroup. Furthermore, LVM normalized per surface area was significantly higher $(+48 \%)$ in $\mathrm{SCI}_{\mathrm{T}} \mathrm{vs} \mathrm{SCI}_{\mathrm{U}}$ subgroup $(P=0.01$ in the pairwise comparison at the post-hoc test). Such positive trend $(+5 \%)$ was observed also between the $\mathrm{ABL}_{\mathrm{T}}$ and the $\mathrm{ABL}_{\mathrm{U}}$ subgroups although it did not reach statistical significance (pairwise comparison at the post-hoc test).

Conversely, EDD and EDV were unchanged in $\mathrm{SCI}_{\mathrm{T}}$ vs $\mathrm{SCI}_{\mathrm{U}}$ subjects, whereas in $\mathrm{ABL}_{\mathrm{T}}$ subjects $\mathrm{EDV}$ was higher than in $\mathrm{ABL}_{\mathrm{U}}$ subjects $(P=0.006$ in the pairwise comparison at the post-hoc test).

Exercise test. The maximal velocity achieved on the wheelchair ergometer, the peak $\mathrm{O}_{2}$ consumption $\left(\mathrm{pVO}_{2}\right)$ and the resting and peak heart rate (HR) in the paraplegic group, stratified according to training status, are shown in Table 3. Significantly higher maximal velocity $(+52 \%)$ and peak $\mathrm{VO}_{2}(+63 \%)$ were observed in the $\mathrm{SCI}_{\mathrm{T}}$ compared with the $\mathrm{SCI}_{\mathrm{U}}$ subgroup. Resting HR was significantly lower in $\mathrm{SCI}_{\mathrm{T}}$ subgroup $(-13 \%)$, whereas peak $\mathrm{HR}$ was not different between subgroups.

None of the echocardiographic parameters significantly correlated with peak oxygen uptake, except for the aortic flow velocity, which showed a significant positive relationship with peak $\mathrm{VO}_{2}$ (Figure 1) in SCI subjects, independently from the training status. Finally, although not reaching statistical significance $(P=0.07)$, a positive trend was observed between peak oxygen consumption and normalized LVM (Figure 2) in the pooled data of paraplegic subjects.

\section{DISCUSSION}

The main finding of this study is that, despite some differences in LV dimensions and function, SCI individuals have similar training response as ABL subjects. A regimen of regular aerobic physical activity may therefore positively change heart morphology and function in paraplegics, thus limiting their cardiovascular risk.

In healthy subjects the end-diastolic dimensions are closely related to preload and venous compliance, and their increase with aerobic training is related to an increased stroke volume. We observed lower end-diastolic dimensions of the left ventricle in paraplegics compared with $A B L$ subjects, which suggests a compromise that may result in a lower stroke volume and therefore a higher HR when exercising or working: this is similar to what was observed after prolonged bed rest. ${ }^{16}$ In SCI patients, the chronic cardiovascular deconditioning due to prolonged wheelchair permanence and the reduced venous return due to the sublesional (that is, splanchnic and lower limbs vasculature) blood pooling may be among the leading causes for this LV atrophy. ${ }^{6}$ However, in this study the IVST was surprisingly higher in paraplegic subjects (both trained and untrained) compared with healthy indivi-

Table 3 Maximal velocity achieved on the wheelchair ergometer, peak $\mathrm{O}_{2}$ consumption $\left(\mathrm{VO}_{2}\right)$, resting and peak heart rate in the paraplegic group divided for training status

\begin{tabular}{lccc}
\hline & $S$ & $S C l_{U}$ & $\mathrm{P}$ \\
\hline Maximal velocity, $\mathrm{km} \mathrm{h}^{-1}$ & $4.73 \pm 0.98$ & $7.20 \pm 1.30$ & 0.001 \\
Peak VO $_{2}, \mathrm{ml} \mathrm{min}^{-1} \mathrm{~kg}^{-1}$ & $13.3 \pm 3.3$ & $21.8 \pm 4.8$ & 0.001 \\
Resting heart rate, b.p.m & $77 \pm 10$ & $67 \pm 7$ & 0.05 \\
Peak heart rate, b.p.m & $140 \pm 19$ & $150 \pm 16$ & $\mathrm{NS}$ \\
\hline
\end{tabular}

Abbreviations: b.p.m., beats per minute; NS, not significant; $\mathrm{SCl}_{\mathrm{T}}$, aerobically trained spinal cord injury subjects; $\mathrm{SCl}_{U}$, aerobically untrained spinal cord injury subjects. Data are mean \pm s.d. $P$, significance value from unpaired Student's $t$ test.

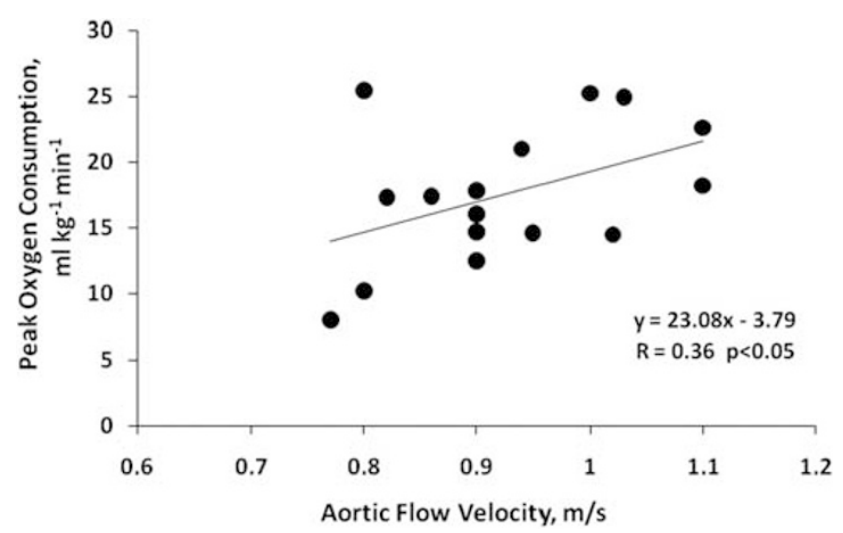

Figure 1 Relationship between aortic flow velocity and maximal oxygen uptake in the groups of paraplegic subjects.

Table 2 Echocardiographic parameters divided for pathology and training status

\begin{tabular}{|c|c|c|c|c|c|c|c|}
\hline $\mathrm{EDD}, \mathrm{mm}$ & $41.4 \pm 5.3$ & $46.6 \pm 5.1$ & $46.0 \pm 5.8$ & $48.7 \pm 5.1$ & 0.040 & NS & NS \\
\hline IVST, mm & $8.6 \pm 0.8$ & $10.2 \pm 1.1$ & $8.3 \pm 1.9$ & $8.6 \pm 0.7$ & 0.020 & 0.014 & 0.001 \\
\hline EDV, ml & $80.4 \pm 18.7$ & $72.6 \pm 21.7$ & $108.9 \pm 30.2$ & $125.0 \pm 20.5$ & 0.001 & NS & 0.009 \\
\hline$E F, \%$ & $61.4 \pm 4.6$ & $60.1 \pm 8.8$ & $67.4 \pm 5.4$ & $63.6 \pm 4.8$ & 0.030 & NS & NS \\
\hline LVM, $\mathrm{g} \mathrm{m}^{-2}$ & $56.3 \pm 17.5$ & $83.1 \pm 15.7$ & $74.0 \pm 16.2$ & $78.0 \pm 14.1$ & NS & 0.014 & NS \\
\hline$E / A$ & $1.64 \pm 0.80$ & $1.49 \pm 0.30$ & $1.59 \pm 0.47$ & $1.70 \pm 0.34$ & NS & NS & NS \\
\hline IVRT, ms & $107.9 \pm 17.7$ & $100.6 \pm 15.7$ & $54.6 \pm 13.8$ & $57.7 \pm 9.9$ & 0.001 & NS & NS \\
\hline
\end{tabular}

Abbreviations: $A$, peak atrial inflow velocity; $A B L_{T}$ aerobically trained able-bodied subjects; $A B L_{U}$, aerobically untrained able-bodied subjects; $E$, peak early inflow velocity; EDV, end-diastolic volume; EDD, end-diastolic diameter; EF, ejection fraction; IVST, intra-ventricular septum thickness; IVRT, iso-volumic relaxation time; LVEDD, left ventricular end-diastolic diameter; LVM, left ventricular mass; NS, not significant; PWT, posterior wall thickness; SCI, spinal cord injuries; SClu, aerobically untrained spinal cord injury subjects. Data are mean \pm s.d. The last three columns show the $P$ values estimated by the factorial ANOVA for the effects of lesion, training and their interaction. 


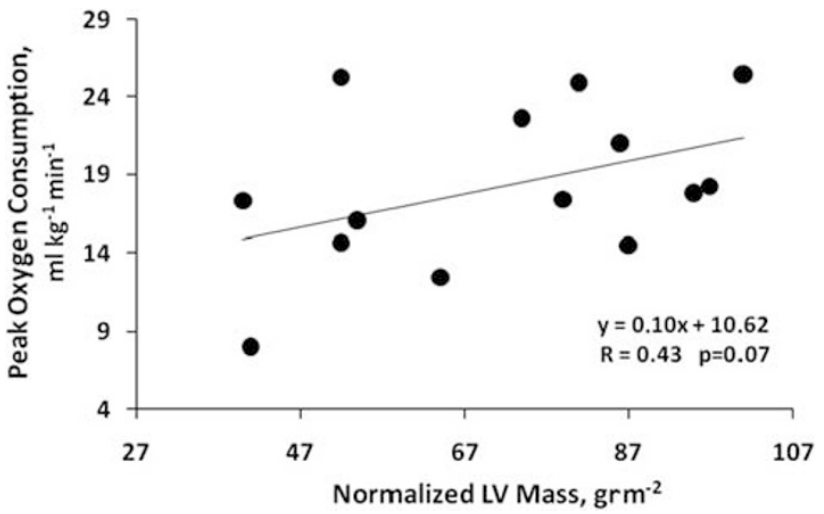

Figure 2 Relationship between normalized LV mass and maximal oxygen uptake in the groups of paraplegic subjects.

duals, and there was also a trend toward an increased PWT $(P=0.13)$. IVST and PWT are usually increased in endurance athletes, as a response of symmetrical cardiac hypertrophy; therefore, their higher values in both trained and untrained paraplegics were unexpected. However, as recently proposed by Matos-Souza et al., ${ }^{4}$ this may suggest that the chronically reduced venous return may have been compensated by a subsequent activation of the hormonal regulatory system, such as the renin-angiotensin-aldosterone system, in order to maintain blood pressure. This, in turn, may have stimulated LV remodeling, increasing LV wall thickness. Interestingly, such effect seems not to occur during the ABL individual's long-term adaptation to training, as suggested by the significance of the interaction term of factorial analysis of variance (Table 2): IVST and PWT increased in SCI subjects only. In addition, EDV tended to decrease in SCI and to increase in ABL subjects (with significant interaction), suggesting probable different mechanisms of adaptation to the training stimulus, which deserves further research. Other previous findings suggest a higher neuro-hormonal influence on the cardiovascular control of SCI subjects. ${ }^{15}$ It is possible that these neuro-hormonal changes (as an increased norepinephrine level or an activation of the renin-angiotensin-aldosterone system), coupled with the typical blood pressure instability, ${ }^{18}$ contribute to the increased cardiovascular risk that characterizes paraplegic individuals.

The diastolic function, as assessed by $E, A$ and $E / A$ ratio, was not significantly impaired in our SCI individuals. These data are consistent with those obtained by Eysmann et al. ${ }^{19}$ by conventional echocardiography, whereas more recently Matos-Sousa et al. ${ }^{4}$ demonstrated a lower early diastolic filling in a group of paraplegics compared with ABL subjects. Interestingly, we reported a higher IVRT in our SCI individuals, which may suggest some difficulties in the very early diastolic filling. Maybe, the possible reduction of LV compliance in paraplegics may have been compensated by an increased isovolumic relaxation time in order to fill the LV adequately, without compromising the subsequent diastolic filling.

Another possible consequence of the impaired venous return in SCI individuals is that stroke volume cannot be adequately increased during incremental exercise. Indeed, as previously demonstrated by Hopman et al., ${ }^{6}$ stroke volume is significantly reduced in paraplegics either at maximal or at submaximal working level (about $-20 \%$ and $-25 \%$, respectively, at $40 \%$ and $60 \%$ of the maximal power output) during an incremental arm-cranking test, compared with ABL subjects. This finally limits the maximal cardiac output and therefore the maximal $\mathrm{VO}_{2}$ measured in SCI people. Our data confirm this hypothesis as, on average, the maximal oxygen uptake of the trained paraplegic subgroup only halved the average value commonly found in aerobically trained ABL people. However, the maximal oxygen uptake was significantly higher and the resting $\mathrm{HR}$ was significantly lower in $\mathrm{SCI}_{\mathrm{T}}$ vs $\mathrm{SCI}_{\mathrm{U}}$ subgroup, demonstrating the positive effects of long-term endurance training on the whole cardiovascular function and a shift toward the parasympathetic predominance of HR control, which can be typically observed in aerobically trained athletes.

Besides training status, we noticed a positive and significant relationship between aortic flow velocity, which can be considered to be a surrogate marker of stroke volume, and peak oxygen uptake in the SCI groups. This suggests that even though in paraplegics the aerobic performance may be influenced by the reduction of stroke volume induced by the sublesional blood pooling, such inability appears to be partially compensated by physical training (Figure 1). In addition, the LVM normalized per body surface area was significantly increased in the $\mathrm{SCI}_{\mathrm{T}}$ compared with the $\mathrm{SCI}_{\mathrm{U}}$ subgroup, and there was a clear trend, although the statistical regression was just below the significance limits, between LVM and peak $\mathrm{VO}_{2}$ (Figure 2). It is acknowledged the LVM is increased by long-term endurance training, and that it represents an independent predictor of maximal work capacity. ${ }^{20}$ Therefore, our findings suggest that aerobic training is able to induce a physiological ventricular hypertrophy even in SCI people. These last data are in agreement with other previous results on the effect of endurance training on oxygen uptake in paraplegics, ${ }^{21}$ and of high-intensity interval training on peak stroke volume in SCI subjects. ${ }^{8}$ In addition, these results parallel those of Dorfman et al., ${ }^{16}$ who showed that the cardiac atrophy that follows the prolonged bed rest can be reversed by training. Finally, although Gates et al. ${ }^{9}$ described only small adaptations of left ventricle to aerobic training, they, however, reported a trend toward an increase in LV mass in SCI athletes, which is in line with the present findings.

In conclusion, this study showed a reduced diastolic filling capacity, an altered heart morphology similar to that of the deconditioned heart in SCI patients with respect to ABL subjects. However, in trained paraplegics, the heart seemed to positively adapt to training, as normalized heart mass and LV wall thickness were both increased: these changes persisted after 5-year training, and parallel those observed in ABL individuals.

Therefore, despite some possible limitations in venous return, aerobic training in SCI individuals seems to promote a physiological cardiac hypertrophy, which may reverse the pathological LV atrophy typically occurring after SCI. Such heart adaptations are similar to those found in ABL subjects. This may be relevant from a clinical point of view, as aerobic training may contribute toward significantly reducing the cardiovascular risk, which is known to be higher in SCI people. ${ }^{22}$

\section{STUDY LIMITATIONS}

This is a case-control study: longitudinal designs would have been preferable in determining the effects of training on heart structure. In addition, we cannot exclude that the small sample size of our study groups could have affected data generalizability.

The heart's dimensional and functional measures were obtained from conventional trans-thoracic echocardiography: perhaps the more recent spectral techniques in tissue Doppler imaging may have added further results, especially on diastolic function.

We did not perform the incremental test in ABL subjects, because they were not used for the wheelchair propulsion on the wheelchair rolling ergometer. Thus, the results of such tests could not be easily compared between the ABL and SCI groups. Finally, all the ABL athletes enrolled in this study had a prevalent use of lower limbs during their training, whereas the trained paraplegics used upper 
limbs during training. Although we consider this aspect of minor relevance for heart adaptation to training, we cannot exclude that this may have produced unpredictable result.

\section{DATA ARCHIVING}

There were no data to deposit.

\section{CONFLICT OF INTEREST}

The authors declare no conflict of interest.

\section{ACKNOWLEDGEMENTS}

We sincerely thank Dr Miriam Longaretti for her valuable contribution to the organization of the study.

1 Pavlik G, Major Z, Varga-Pinter B, Jeserich M, Kneffel Z. The athlete's heart Part I (Review) Acta Physiol Hung 2010; 97: 337-353.

2 Calderon FJ, Diaz V, Peinado AB, Benito PJ, Maffulli N. Cardiac dimensions over 5 years in highly trained long-distance runners and sprinters. Phys Sportsmed 2010; 38: 112-118.

3 de Groot PC, van Dijk A, Dijk E, Hopman MT. Preserved cardiac function after chronic spinal cord injury. Arch Phys Med Rehabil 2006; 87: 1195-1200.

4 Matos-Souza JR, Pithon KR, Oliveira RT, Téo FH, Blotta MH, Cliquet Jr A et al. Altered left ventricular diastolic function in subjects with spinal cord injury. Spinal Cord 2011; 49: 65-69.

5 Dela F, Mohr T, Jensen CM, Haahr HL, Secher NH, Biering-Sørensen F et al. Cardiovascular control during exercise: insights from spinal cord-injured humans. Circulation 2003; 107: 2127-2133.

6 Hopman MT, Oeseburg B, Binkhorst RA. Cardiovascular responses in paraplegic subjects during arm exercise. Eur J Appl Physiol Occup Physiol 1992; 65: 73-78.

7 Merati G, Sarchi P, Ferrarin M, Pedotti A, Veicsteinas A. Paraplegic adaptation to assisted-walking: energy expenditure during wheelchair versus orthosis use. Spinal Cord 2000; 38: 37-44
8 Brurok B, Helgerud J, Karlsen T, Leivseth G, Hoff J. Effect of aerobic high-intensity hybrid training on stroke volume and peak oxygen consumption in men with spinal cord Injury. Am J Phys Med Rehabil 2011; 90: 407-414.

9 Gates PE, Campbell IG, George KP. Absence of training-specific cardiac adaptation in paraplegic athletes. Med Sci Sports Exerc 2002; 34: 1699-1704.

10 Sahn DJ, DeMaria A, Kisslo J, Weyman A. Recommendations regarding quantitation in M-mode echocardiography: results of a survey of echocardiographic measurements. Circulation 1978; 58: 1072-1083.

11 Devereux RB, Reichek N. Echocardiographic determination of left ventricular mass in man. Anatomic validation of the method. Circulation 1977; 55: 613-618.

12 Ishida Y, Meisner JS, Tsujioka K, Gallo JI, Yoran C, Frater RW et al. Left ventricular filling dynamics: influence of left ventricular relaxation and left atrial pressure. Circulation 1986; 74: 187-196.

13 Hartung GH, Lally DA, Blancq RJ. Comparison of treadmill exercise testing protocols for wheelchair users. Eur J Appl Physiol Occup Physiol 1993; 66: 362-365.

14 Frey GC, McCubbin JA, Dunn JM, Mazzeo RS. Plasma catecholamine and lactate relationship during graded exercise in men with spinal cord injury. Med Sci Sports Exerc 1997; 29: 451-456.

15 Craig CL, Marshall AL, Sjöström M, Bauman AE, Booth ML, Ainsworth BE et al. International physical activity questionnaire: 12-country reliability and validity. Med Sci Sports Exerc 2003; 35: 1381-1395.

16 Dorfman TA, Levine BD, Tillery T, Peshock RM, Hastings JL, Schneider SM et al. Cardiac atrophy in women following bed rest. J Appl Physiol 2007; 103: 8-16.

17 Schmid A, Huonker M, Stahl F, Barturen JM, König D, Heim M et al. Free plasma catecholamines in spinal cord injured persons with different injury levels at rest and during exercise. J Auton Nerv Syst 1998; 68: 96-100.

18 Castiglioni P, Di Rienzo M, Veicsteinas A, Parati G, Merati G. Mechanisms of blood pressure and heart rate variability: an insight from low-level paraplegia. Am J Physiol Regul Integr Comp Physiol 2007; 292: R1502-R1509.

19 Eysmann SB, Douglas PS, Katz SE, Sarkarati M, Wei JY. Left ventricular mass and diastolic filling patterns in quadriplegia and implications for effects of normal aging on the heart. Am J Cardiol 1995; 75: 201-203.

20 Steding K, Engblom H, Buhre T, Carlsson M, Mosén H, Wohlfart B et al. Relation between cardiac dimensions and peak oxygen uptake. J Cardiovasc Magn Reson 2010; 12: 8.

21 Fukuoka Y, Nakanishi R, Ueoka H, Kitano A, Takeshita K, Itoh M. Effects of wheelchair training on VO2 kinetics in the participants with spinal-cord injury. Disabil Rehabil Assist Technol 2006; 1: 167-174.

22 Wahman K, Nash MS, Lewis JE, Seiger A, Levi R. Cardiovascular disease risk and the need for prevention after paraplegia determined by conventional multifactorial risk models: the Stockholm spinal cord injury study. J Rehabil Med 2011; 43: 237-242. 